\title{
Género discursivo, sintaxis y lenguaje académico: Dimensiones comunicativas y cognitivas del desarrollo tardío del lenguaje
}

\author{
Alejandra MENESES ARÉVALO \\ Universidad Católica de Chile \\ Facultad de Educación \\ amenesea@uc.cl \\ Maili OW GONZÁLEZ \\ Universidad Católica de Chile \\ Facultad de Educación \\ mow@uc.cl
}

Recibido: marzo 2011

Aceptado: marzo 2012

\begin{abstract}
RESUMEN
El desarrollo sintáctico tardío ha sido estudiado preferentemente desde una visión estructuralista del lenguaje, describiendo el surgimiento de distintos tipos de estructuras sintácticas a lo largo de la escolaridad. La escuela por su parte, dependiendo de las tradiciones instruccionales ha desplazado la enseñanza de una gramática explícita para dar paso a un enfoque comunicativo en el que el papel de esta aún no se logra afianzar. En este artículo se realiza una revisión crítica de los distintos supuestos sobre los que se fundamentan las investigaciones sobre desarrollo de la sintaxis para realizar una propuesta teórica de articulación entre discurso y sintaxis desde un enfoque socio-discursivo. Basándose en la visión de Bajtín (1982) del lenguaje como actividad social y su propuesta sobre géneros discursivos, se aborda la escuela como un marco de interacción con exigencias comunicativas y cognitivas propias. De esta manera, la escuela se caracteriza por exponer a los estudiantes a 'géneros discursivos secundarios' y a un nuevo tipo de conocimiento: 'los conceptos científicos'. Por tanto, el concebir el lenguaje como actividad social no implica solo poner en el centro de la reflexión la dimensión comunicativa del lenguaje sino también el vínculo con la actividad cognitiva.
\end{abstract}

Palabras clave: Géneros discursivos secundarios, desarrollo sintáctico, lenguaje académico, conceptos científicos. 


\title{
Genre discursif, syntaxe et langage académique: Dimensions communicatives et cognitives du développement tardif du langage
}

\section{RÉSUMÉ}

Le développement syntaxique tardif a de préférence été étudié depuis une vision structuraliste du langage, par la description de l'apparition de différents types de structures syntaxiques au long de la scolarité. Dépendante des traditions instructionnelles, l'école a, quant à elle, déplacé l'enseignement d'une grammaire explicite pour donner place à une approche communicative dans laquelle le rôle de cette dernière n'a pas encore pu être consolidé. Dans cet article, nous réalisons un examen critique des différents supposés sur lesquels reposent les recherches sur le développement de la syntaxe, afin de faire une proposition théorique d'articulation entre discours et syntaxe depuis une approche sociodiscursive.

Partant de la vision de Bajtin (1982) du langage comme activité sociale et de sa proposition sur les genres discursifs, nous abordons l'école comme un cadre d'interaction doté d'exigences communicatives et cognitives propres. Ainsi, l'école se caractérise par le fait qu'elle expose aux élèves les "genres discursifs secondaires" et un nouveau type de connaissance: "les concepts scientifiques". C'est pourquoi concevoir le langage comme une activité sociale implique de mettre au centre de la réflexion non seulement la dimension communicative du langage mais aussi le lien avec l'activité cognitive.

Mots-clé: Genres discursifs secondaires - développement syntaxique - langage académique - concepts scientifiques

\section{Genre, Syntax and Academic Language: Communicative and Cognitive Dimensions of Later Language Development}

\begin{abstract}
The later syntactic development has been studied mainly form a structuralist view of language, describing the emergence of different types of syntactic structures throughout the school years. The school in turn, depending on the traditions of teaching has shifted the explicit teaching of grammar to move to a communicative approach. In this paper, a critical review of different assumptions based on research of syntactic development is carried out to propose a theoretical link between discourse and syntax form a socio-discursive approach. Based on Bakhtin's vision (1982) of the language as a social activity and his Speech Genre Theory, the school is conceived as a new framework of interaction with communicative and cognitive demands of its own. Thus, the school is characterized by exposing student to 'secondary speech genres' and a new type of knowledge 'the scientific concepts'. Therefore, the language's conception as a social activity implies not only to put in the center of reflection the communicative dimension of language but also the link with the cognitive activity.
\end{abstract}

Key words: Secondary genres-syntax development-academic language- scientific concepts. 
SUMARIO: 1. Introducción. 2. Género discursivo, discurso y sintaxis: dimensiones comunicativas y cognitivas del desarrollo del lenguaje. 3. El desarrollo sintáctico tardío: desde las estructuras a las funciones. 4. La escuela: del código escrito al lenguaje académico. 5. Conclusiones. 6. Referencias bibliográficas.

\section{INTRODUCCIÓN}

Desde la reforma educativa chilena de los años 90, la gramática ha sido desplazada del ámbito escolar a un segundo plano, pues la enseñanza de la lengua ha dado, más bien, un giro hacia el desarrollo de la competencia comunicativa que amplía los componentes de enseñanza y posiciona a la gramática en un marco mayor $\mathrm{y}$, sin duda, secundario. De este modo, se ha buscado modificar, en numerosas ocasiones sin buenos resultados, una serie de prácticas pedagógicas de reconocimiento y análisis de diferentes estructuras gramaticales aisladas de contextos comunicativos y cuyos resultados estaban más vinculados con el desarrollo de habilidades lógicas que con un incremento de la comunicación. No obstante, este desplazamiento de la gramática no ha logrado un enfoque efectivo para el desarrollo del lenguaje académico oral y escrito que se espera ocurra durante la escolaridad (Ravid \& Tolchinsky, 2002). De esta forma, en las aulas y en los textos escolares de estudio ha convivido simbióticamente un enfoque tradicional de enseñanza de la lengua basado en el sistema, con una propuesta comunicativa y discursiva, sin establecer vínculos explícitos con el desarrollo de las habilidades lingüísticas que necesitan los estudiantes (Medina, 2002). Como plantea Álvarez (2006), la enseñanza de la lengua se ha encontrado en una tensión entre el sistema y el uso comunicativo, predominando, al menos en el discurso pedagógico oficial, este último enfoque. Sin embargo, enseñar a leer, a escribir y a expresarse oralmente implica no solo un dominio progresivo de estructuras textuales, sino también de recursos léxico-gramaticales vinculados a estas. Por este motivo es necesario describir dichos recursos léxico-gramaticales vinculados a los formatos textuales en términos de trayectorias de desarrollo y aprendizaje en los niños y adolescentes. Esto contribuirá a aumentar las habilidades comunicativas de los estudiantes.

Ahora bien, Byrnes y Wasik (2009) señalan que el término 'gramática' es entendido de manera distinta por los estudios del desarrollo del lenguaje y por los contextos instruccionales de la lectura y la oralidad. Este divorcio ha provocado que muchos esfuerzos de didáctica de la lengua sean estériles, encontrándonos así ante un tema no resuelto. En este sentido, cabe preguntarse ¿qué rol juega la gramática en el aprendizaje de la lectura y escritura? ¿De qué manera se van complejizando ciertas estructuras sintácticas en la producción oral de narraciones y explicaciones a través de la escolaridad? ¿Cómo se vinculan estas estructuras en la oralidad con la capacidad de producir textos escritos? Estas son algunas de las preguntas de investigación del proyecto FONDECYT 1100600, "El desarrollo sintáctico tardío en la oralidad y las modalidades discursivas: hacia una visión 
funcional del desarrollo lingüístico en la edad escolar", en el que se inserta el presente trabajo.

Dentro de este marco, en este artículo se desarrolla una propuesta teórica de articulación entre sintaxis y discurso desde una mirada socio-discursiva con el fin de poder demostrar la relevancia de afrontar una empresa de descripción del desarrollo sintáctico tardío de la oralidad y su relación con el emerger del lenguaje académico, propio de la escolaridad. El artículo se inicia con una revisión crítica de los aportes de la teoría de los géneros discursivos en Bajtín y su proyección al plano cognitivo. Posteriormente, se extiende esta reflexión al plano sintáctico y a la necesidad de abordar el desarrollo de esta dimensión del lenguaje desde una aproximación socio-discursiva, para finalizar con la relación entre escolaridad y desarrollo del lenguaje académico.

\section{GÉNERO DISCURSIVO, DISCURSO Y SINTAXIS: DIMENSIONES COMUNICATIVAS Y COGNITIVAS DEL DESARROLLO DEL LENGUAJE}

Desde el punto de vista histórico, la concepción lógico-gramatical tal como plantea Bronckart (2008) establece la independencia entre los procesos de pensamiento y los procesos de significación semiótica; es decir, los signos de la lengua son concebidos como formas de expresión de determinadas unidades previas de pensamiento. Por lo tanto, habría un lenguaje universal que daría forma a las operaciones cognitivas donde las lenguas y los diversos contextos socioculturales no juegan un rol en la conformación de estas. El estructuralismo sausseriano, en cambio, puso en el centro de la reflexión a las lenguas históricas en cuanto sistemas de signos distinguiéndolas de los eventos comunicativos. Sin embargo, para Bajtín (1982) la distinción sausseriana entre lengua y habla es engañosa; pues no existe un sistema estable de formas lingüísticas que puedan ser estudiadas independientemente de su contexto, olvidando que estos son producciones socio-históricas y subjetivas. La lengua como sistema es una abstracción que no da cuenta del funcionamiento del lenguaje, en tanto proceso social, discursivo e interactivo entre los hablantes de una comunidad o esfera social (Bajtín, 1982). Si algo ha de ser estudiado es el habla: instrumento de comunicación entre seres vivos encarnados en un tiempo y en un espacio, cambiante y atravesado por la historia. Asimismo, Bajtín y su círculo cuestionan un aspecto fundamental de la distinción saussureana: el enfrentamiento de lo social y lo individual (Emerson, 1993). Si la lingüística estructural se ha centrado en el estudio de lo sistemático, en tanto que independiente de las prácticas lingüísticas individuales, situadas e históricas, Bajtín propondrá una metalingüística que evite una visión esencialista del lenguaje (Stewart, 1993) y que estudie los aspectos de la vida de las palabras; palabras como enunciaciones en contexto y en diálogo. La 
clave no están solo en lo que las palabras "refieren", sino en las acciones que desencadenan, lo que acerca su propuesta a los terrenos de la pragmática.

Bajtín propone ir más allá del estudio de los niveles léxico y gramatical y considera que las unidades de análisis del sistema de la lengua (fonema, morfema, palabra y oración) no dan cuenta de su potencial comunicativo; de esta forma, propone una concepción del lenguaje dinámica y ligada a las interacción social. En la visión metalingüística de Bajtín, la unidad mínima de comunicación es el 'enunciado', entendido como un acto concret, con componentes sociales, pero de realización siempre individual; es un complejo material -sonoro, articulatorio, visual- que forma parte de la realidad social, pero que siempre es fruto de un sujeto concreto. Vale la pena, en todo caso, aclarar que el término 'enunciado' utilizado por Bajtín se aproxima bastante al concepto de 'discurso' propuesto por el análisis del discurso. Charaudeau y Maingueneau (2005) distinguen entre las distintas significaciones de este concepto polisémico; por una parte, se opone a 'lengua', definido este último como sistema de posibilidades; en cambio, 'discurso' se refiere, más bien, al uso de la lengua en un determinado contexto de comunicación. Al determinar que la unidad mínima de uso de la lengua es el enunciado, la propuesta bajtiniana supone comprender el lenguaje como actividad situada y configuradora de las esferas sociales de comunicación. Respecto de la dimensión cognitiva, al no ser las significaciones reflejo de estructuras de pensamientos previas sino, más bien, que al ser creadas mediante la actividad lingüística en los diferentes contextos de interacción social, la interiorización de estos discursos externos, de carácter social produce un discurso interno de carácter subjetivo, dialógico y epistémico (Bronckart, 2008; Voloshinov \& Bajtin, 1998).

En un nivel menos individual, en tanto que no depende del hablante, Bajtín introduce el concepto de 'género discursivo'. "Cada enunciado por separado es, por supuesto, individual, pero cada esfera de la lengua elabora sus tipos relativamente estables de enunciados" (Bajtín, 1982, 248). Los géneros discursivos, usando la terminología de Saussure, se sitúan entre la lengua y el habla. El hablante los elige -no los crea-, le son dados casi del mismo modo que la comunidad nos dona una determinada lengua. A la vez, dan cuenta de la práctica individual, situada e histórica. Cada tiempo, cada cultura, cada manifestación lingüística del hombre, más o menos institucionalizada, posee sus propios géneros discursivos. Así, Bajtín (1982) distingue entre enunciado (discurso) entendido como la acción que realiza un sujeto utilizando el lenguaje como herramienta comunicativa en un contexto particular y género discursivo como un escenario de actividad que produce una serie relativamente estable de formas discursivas que permiten la comunicación en una esfera de la vida social: "si no existieran los géneros discursivos y si no los domináramos, si tuviéramos que irlos creando cada vez dentro del proceso discursivo, libremente y por primera vez cada enunciado, la comunicación discursiva habría sido casi imposible" (Bajtín, 1982, 268). 
De esta manera, Voloshinov y Bajtín (1998) proponen un tipo de análisis de los discursos que considera las condiciones de producción y la interacción social de estos; el punto de partida de las significaciones de cualquier evento comunicativo dice relación con la situación de comunicación y con los participantes de esta y, por ende, las elecciones de las formas textuales y lingüísticas dependen de este marco comunicativo. Bronckart $(2008,9)$ puntualiza

Desde esta perspectiva, Voloshinov propone entonces un programa metodológico, que se caracteriza por un proceso de análisis descendiente de la actividad lingüística concretada en el discurso dialógico: en primer lugar, plantea analizar las condiciones y los procesos de interacción social (o incluso, las formas materiales precisas de la expresión de la psicología del cuerpo social (Voloshinov, 1977), es decir, las formas de las actividades humanas con las cuales se articulan y en las que se ubican las producciones del lenguaje; luego se deben analizar la estructura global y las propiedades de estas producciones del lenguaje (los "tipos o géneros de textos/discursos" que semiotizan las interacciones) $\mathrm{y}$, finalmente, analizar las estructura interna de esos textos/discursos, o los niveles allí implicados de organización de los signos: partes del texto, estructuras semántico-sintácticas, valores de unidades mínimas o de las palabras.

Se puede observar, entonces, cómo para Bajtín las formas lingüísticas construcciones sintácticas y unidades léxicas- son concebidas en tanto repertorio a disposición del sujeto que construye un discurso con el fin de poder expresar y representar de manera efectiva, subjetiva y creativa lo que se busca comunicar (Bazerman, 2005). Bajtín (1982, 273) precisa

la oración como unidad de la lengua, igual que la palabra, no tiene autor. No pertenece a nadie, como la palabra, y tan sólo funcionando como un enunciado completo llega a ser la expresión de la postura individual de hablante en una situación concreta de la comunicación discursiva.

La propuesta bajtiana de construcción de significación y la relación con la sintaxis puede, por tanto, representarse como lo muestra la Figura 1.

Figura 1: Propuesta bajtiniana sobre género discursivo, discurso y formas lingüísticas

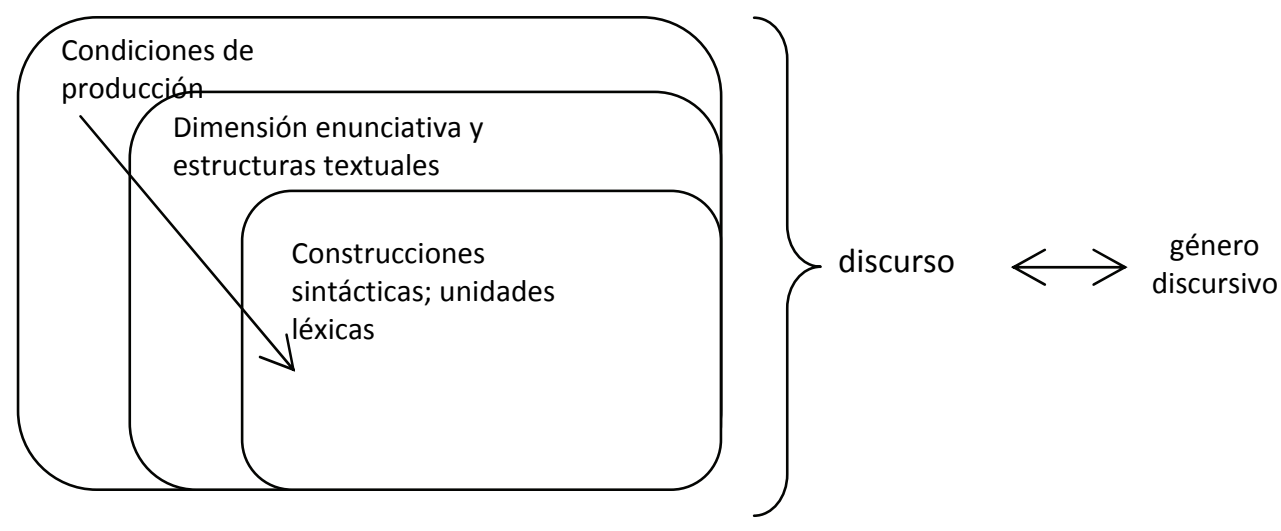


Por lo tanto, las formas lingüísticas son constitutivas de la actividad de lenguaje; de ahí la necesidad de poder establecer vínculos explícitos entre discursos, estructuras textuales, instancias enunciativas y la materialidad lingüística que los conforman y los transforman.

\section{EL DESARROLLO SINTÁCTICO TARDÍO: DESDE LAS ESTRUCTURAS A LAS FUNCIONES}

Estudiar el desarrollo sintáctico implica, desde el punto de vista teórico, contestar a dos preguntas clave tal como lo plantean Byrnes y Wasik (2009). Por una parte, ¿en qué consiste el conocimiento gramatical? $\mathrm{O}$ ¿qué quiere decir que los niños posean un conocimiento gramatical?; y, por otra parte, desde una mirada ontogenética del lenguaje, ¿qué permite que dicho conocimiento gramatical cambie a lo largo del tiempo? Dependiendo de la teoría en la que se sustentan las investigaciones realizadas, pueden, entonces, entenderse adecuadamente las categorías escogidas para ser descritas, los resultados obtenidos y los alcances de los estudios. Desde una perspectiva innatista de tipo chomskiana, se han descrito estructuras lingüísticas en cuanto parte de un sistema programado que emerge en el niño al nacer y que se consolida hacia los cincos años. Byrnes y Wasik (2009) argumentan que de ser adecuadas las explicaciones innatistas, la consolidación del desarrollo gramatical hacia los cinco años en término de adquisición de categorías, parámetros e incremento de la memoria del trabajo, ¿cómo se puede explicar que tanto padres como educadores se preocupen de que todos los niños hayan afianzado una serie de estructuras gramaticales antes de comenzar el aprendizaje formal de la lectura y escritura? Por otra parte, ¿cómo el conocimiento gramatical crece más allá de los cinco años en los contextos de escolarización y de aprendizaje de discursos ligados a las diferentes disciplinas escolares?

Por lo tanto, preguntarse por el desarrollo sintáctico desde la perspectiva del desarrollo tardío del lenguaje conlleva establecer vínculos entre el conocimiento gramatical y los procesos de enseñanza y aprendizaje de la alfabetización y del lenguaje propio de la escuela. Como señala Nippold (2004), el desarrollo tardío del lenguaje no puede ser explicado solo por el conocimiento lingüístico sin situarlo en el contexto de la alfabetización, la escolaridad y el desarrollo de la competencia metalingüística. En consecuencia, implica hacerse cargo del incremento de ciertos conocimientos gramaticales más allá de los 5 años, cuestión no abordada por la teorías innatistas.

En esta línea, una de las nociones clave para el estudio de la complejidad sintáctica vinculada a la escolarización ha sido la 'unidad T', establecida por Hunt (1965) en su estudio sobre producciones escritas de estudiantes norteamericanos. Dichas unidades son definidas como estructuras intermediarias entre la oración y la cláusula, por ende, es una noción sensible a captar cláusulas subordinadas y 
coordinadas al interior de una estructura mayor. Las mediciones para determinar la complejidad sintáctica realizadas por Hunt (1968) fueron cinco: 1) promedio de palabras por cláusula (largo de cláusula); 2) promedio de cláusulas por 'unidades T'; 3) promedio de palabras por 'unidad T' (largo de la unidad T); 4) promedio de 'unidades T' por oración (índice de coordinación por cláusula principal); 5) promedio de palabras por oración (largo de oración). Por su parte, Loban (1976) extrapola el concepto de 'unidades $\mathrm{T}$ ' a contextos de producciones orales estableciendo la noción de 'unidad C' (Communication Unit), definida como la cláusula independiente con sus modificadores. De este modo, incluye las predicaciones no verbales altamente productivas en contextos de interacción cara a cara. Dichos estudios permitieron no solo avanzar en la descripción de las estructuras sintácticas a lo largo de la escolaridad sino también construir índices de subordinación que permitan explicar de manera significativa las diferencias entre las producciones de niños y adultos. Sin embargo, estos estudios de corte estructuralista describen la aparición de diferentes formas (cláusulas de relativos, cláusulas sustantivas, cláusulas adverbiales) en la producción oral y escrita de niños y adultos sin establecer vínculos entre tipos de estructuras y funcionamiento de la mismas en la construcción de diferentes tipos de discurso.

En el ámbito chileno, Véliz (1988) se propuso determinar los parámetros de crecimiento sintáctico promedio en el discurso escrito en una muestra de sujetos de $4^{\circ}, 6^{\circ}, 8^{\circ}$ básico, II y IV medio. Para ello utilizó las 'unidades T' de Hunt y construyó una tarea de tipo narrativa, cuyas temáticas decían relación con el recontado de una película y la narración libre de aventuras de un ser extraterrestre. Dicho estudio demostró que a medida que los sujetos avanzan en escolaridad producen textos escritos con cláusulas más extensas y tienden a construir 'unidades T' más complejas por el número de estructuras incrustadas. Veliz concluye que los resultados obtenidos sobre madurez sintáctica son solo válidos para el discurso narrativo, dado el tipo de tarea construida. En efecto, en un estudio posterior realizado por Véliz (1999) se planteó como objetivo determinar si el modo de organización del discurso se vincula con la complejidad sintáctica de los textos. Los resultados mostraron que el discurso argumentativo era el más complejo en término de la extensión de las cláusulas, del número de cláusulas subordinadas y de incrustaciones de estructuras clausulares. En cambio, el discurso narrativo era el que presentaba menor complejidad sintáctica. Los estudios realizados por Valencia, Ávila, Muñoz, Núñez, Echeverría y Véliz. (1991) y Véliz $(1998,1999)$ utilizaron como marco teórico el establecido por Hunt y validaron para la realidad nacional los índices de complejidad sintáctica en el estudio de producciones escritas. Asimismo, cabe destacar que los resultados obtenidos mostraron que el modo de organización del discurso tiene una incidencia significativa en el grado de complejidad sintáctica, sin establecer relaciones entre sintaxis y construcción de los discursos. 
Por otra parte, solo el estudio de Coloma, Peñaloza y Fernández (2007) abordó la complejidad sintáctica en la producción oral de niños mayores de 7 años identificando los tipos de oraciones producidas por 90 sujetos de $3^{\circ}$ y $5^{\circ}$ básico. Los resultados obtenidos mostraron que los sujetos de 10 años utilizan cláusulas sustantivas para relatar discursos referidos; en cambio, los sujetos más pequeños realizan la misma acción discursiva mediante el uso de oraciones yuxtapuestas. Si bien las investigaciones nacionales revisadas han estudiado la complejidad sintáctica en el discurso escrito y oral, el enfoque de dichos trabajos ha sido mayoritariamente de tipo estructural centrándose, más bien, en la identificación y frecuencia de determinadas construcciones sintácticas, sin ahondar en los aspectos discursivos relacionados con la sintaxis.

Surge, entonces, la pregunta sobre la visión de lenguaje y de gramática que sustenta los estudios descritos y sobre la relación entre estructuras gramaticales y madurez sintáctica en estos. Desde una visión estructuralista del lenguaje, aprender una lengua implica dominar progresivamente categorías y construcciones gramaticales. En este sentido, el desarrollo del lenguaje se explica por la exposición y refuerzo de determinadas estructuras (Byrnes \& Wasik, 2009). Sin embargo, para avanzar en el conocimiento lingüístico y su desarrollo, la sola presencia de "una forma lingüística no nos aporta la necesaria información sobre aspectos tan relevantes como la relación entre forma-función en el lenguaje infantil o el conocimiento de los niños sobre cuáles son las nociones "gramaticalizables" en su lengua" (Sebastián, 2003).

Tomasello (2000) plantea que explicar el desarrollo del lenguaje desde un modelo de tipo pragmático o del uso implica partir desde eventos comunicativos en los cuales los sujetos aprenden y usan el lenguaje. Por lo tanto, de sumo interés resulta estudiar las estructuras lingüísticas en cuanto repertorios disponibles que posibilitan a los sujetos responder a diferentes necesidades comunicativas (Berman \& Katzenberger, 2004; Nir \& Berman, 2010; Tomasello, 2000). En esta línea, los estudios funcionalistas dentro del marco del proyecto de descripción lingüística de diferentes lenguas liderado por Berman (Berman \& Katzenberger, 2004; Berman, 2008; Berman \& Nir, 2007; Nir \& Berman, 2010) han establecido una serie de dispositivos analíticos para describir la arquitectura intraclausular como intradiscursiva estableciendo categorías que permiten establecer vínculos entre sintaxis y retórica; asimismo, dichos estudios han emprendido la tarea de describir la complejidad sintáctica en diferentes modalidad de comunicación (oral y escrita), diferente tipo de discurso (narrativo y expositivo) y en diferentes edades (9-10, 12$13,16-17$ y universitarios adultos).

\section{ESCUELA: DEL CÓDIGO ESCRITO AL LENGUAJE ACADÉMICO}

Desde la concepción del lenguaje como actividad humana generadora de significaciones según lo postulado por Bajtín (1982), ¿cuáles son los desafíos a los 
que se ve enfrentado un estudiante al ingresar a la escuela desde el punto de vista comunicativo, lingüístico y cognitivo? ¿Qué caracteriza a la escuela como espacio de comunicación y sociabilización? En general, se asocia la escuela con el espacio para el aprendizaje formal de la lectura y escritura, cuestión de vital importancia en los primeros años de escolaridad. Numerosos debates hay sobre cuál es el método más efectivo para la enseñanza de la lectura; es indudable que la tarea de aprender a leer y escribir implica no solo el conocimiento de las letras sino el uso de estas en un contexto comunicativo nuevo y distinto al de la familia en el cual el niño está acostumbrado a desenvolverse. Como planteaba Vygostky $(1979,178)$ "a los niños debiera enseñárseles el lenguaje escrito, no la escritura de letras". ¿Qué conlleva este aprendizaje del 'lenguaje escrito' descrito desde la propuesta de géneros discursivos bajtinianos?

Dependiendo de la mayor o menor formalización y complejidad de la comunicación, Bajtín (1982) distingue dos grandes grupos de géneros discursivos: primarios y secundarios. Los géneros discursivos primarios se utilizan en la comunicación cotidiana, están menos formalizados, son más simples y mutables, por lo que varían con más frecuencia; generalmente, son orales. Los géneros discursivos secundarios, en cambio, surgen en esferas de la actividad cultural en las que la comunicación está más organizada, es más sofisticada y se ha ido fosilizando en estructuras discursivas complejas. Ejemplos de los géneros secundarios se producen en las esferas de la comunicación artística, científica y académica. Los géneros primarios, según López García (2000), son los habituales y exclusivos en los niños antes de la edad escolar. De acuerdo con Silvestri (2002b), estos géneros poseen estructuras sintácticas paratácticas, con bajos índices de subordinación e incrustación, con una densidad léxica entre 5 y 1200 palabras. Asimismo, se trata en su mayoría de discursos heterogestionados, ofreciendo soporte cognitivo y comunicativo para la construcción textual.

Por otra parte, en los géneros secundarios, de desarrollo tardío, "la complejidad se eleva en todos los planos, por ejemplo, la densidad léxica se eleva típicamente a 3-6 y el índice de subordinación sube a 1-3, con mayor frecuencia de incrustación" (Silvestri, 2002b, 5). Cognitivamente, los géneros primarios y secundarios ponen en juego funciones psicológicas de distinta complejidad, absorbiendo los segundos las necesidades cognitivas de los primeros, principalmente de carácter oral y conversacional. En este sentido, Silvestri (2002b) sostiene que para usar los géneros secundarios los estudiantes deben aprender a construir un discurso de manera monogestionada. "Se trata de una exigencia cognitiva fuerte, porque implica activar información en memoria en ausencia de los aportes del interlocutor y exige mantener la coherencia y la imagen del receptor cuando no hay retroalimentación" (Silvestri, 2002b, 5).

Por este motivo, la autora señala que los géneros secundarios implican un manejo consciente de recursos simbólicos, con presencia de procesos metacognitivos y conciencia en la selección de elementos léxicos, sintácticos y 
discursivos. Se ha asociado los géneros discursivos secundarios con la escritura; sin embargo, es necesario explorar el modo en que son aprendidos los géneros orales secundarios en contextos escolares y su relación con el aprendizaje del lenguaje académico. Es más, ¿cuáles son los recursos sintácticos, léxicos y discursivos que configuran estos géneros orales propios del lenguaje académico y cómo estos repercuten en el aprendizaje de los géneros escritos?

Ahora bien, si retomamos la propuesta vygostkiana sobre la lengua escrita y su relación con la cognición, no queda claro si el aprendizaje de la lengua escrita en sí es lo que lleva al desarrollo del pensamiento o bien es el ingreso al marco institucional educativo lo que lleva a un nuevo escenario de actividad: la enseñanza-aprendizaje de diferentes áreas de conocimiento científico de la realidad (Cubero \& Sánchez, 2002; Silvestri, 2002a, 2002b). Sin embargo, para Vygostky (1979), el nivel de abstracción mayor es posibilitado por el aprendizaje la lengua escrita en cuanto implica utilización de un sistema de signos desvinculados de su contexto comunicativo inmediato. Entonces, ¿qué es lo que lleva al ser humano a un mayor nivel de abstracción y generalización de su pensamiento? La mayoría de los estudios está de acuerdo en que no es suficiente el conocimiento del código escrito para desarrollar el pensamiento; más bien, los procesos de alfabetización están ligados al aprendizaje de diversas prácticas letradas en determinadas comunidades ( Cubero \& Sánchez, 2002; Tolchinsky \& Simó, 2001). Como señalan Cubero y Sánchez $(2002,108)$ "la razón fundamental para tal transformación es que en las escuelas las personas se especializan en un nuevo método para resolver problemas y en un nuevo tipo de conocimientos y conceptos -los conceptos científicos."

Por lo tanto, se puede establecer un diálogo entre el planteamiento vygostkiano sobre el desarrollo de los conceptos científicos y su vinculación con los conceptos cotidianos y la propuesta bajtiana sobre géneros secundarios y primarios. De ahí entonces que este tipo de actividades complejas planteen un desafío cognitivo mayor, pues requieren que el individuo sea capaz de llevar a cabo una actividad metacognitiva consciente en los diversos planos: socio-cultural, discursivo, composicional-textual, léxico y sintáctico para cumplir con un objetivo comunicativo de tipo epistémico. Por lo tanto, se da una relación dialéctica entre prácticas sociales y modos de pensamiento que se materializan a través del lenguaje en cuanto herramienta comunicativa y de conocimiento.

Como plantea Schleppegrell (2001), la escuela, en la mayoría de los casos, no es consciente de los desafíos lingüísticos y cognitivos que exige a los estudiantes. De ese modo, se centra sobre todo en los resultados obtenidos por estos, asumiendo que los estudiantes pueden hacerse cargo de utilizar el lenguaje oral y escrito en contextos académicos sin brindarles soporte y andamiaje para poder enfrentar las exigencias del nuevo contexto comunicativo. Snow y Uccelli (2009) precisan que todo discurso es complejo, sin embargo, la complejidad propia del lenguaje académico radica en el contenido del mensaje y en su estatus epistemológico. De 
ahí, entonces, también la relación entre la escuela y el desarrollo de los conceptos científicos propuestos por Vygostky. Asimismo, resulta de interés el modo en que las autoras describen la complejidad del lenguaje académico no solo en términos de formas lingüísticas propias de la escritura, sino más bien como trayectorias de aprendizaje que van de la oralidad a la escritura, de un registro informal a uno formal y de estructuras narrativas a explicativas. Por lo tanto, cabe preguntarse qué rol juegan las estructuras gramaticales y léxicas en estas trayectorias de aprendizaje con el fin de poder establecer estrategias de enseñanza que permitan no solo el aprendizaje eficaz de la lectura y escritura (decodificar y codificar) sino que permitan al niño poder ingresar de manera satisfactoria al nuevo contexto comunicativo que le brinda la escuela.

\section{CONCLUSIONES}

En este artículo se ha realizado una revisión crítica de los presupuestos sobre los que se fundamentan las investigaciones sobre desarrollo sintáctico tardío y se ha esbozado una propuesta teórica partiendo de los postulados bajtinianos sobre la naturaleza social del lenguaje y la organización de los géneros discursivos en primarios y secundarios. Este marco conceptual permite poner en el centro de la reflexión al lenguaje como actividad creadora de significaciones no solo en plano social sino también en el plano cognitivo. De esta manera, estudiar la complejidad sintáctica en relación con el desarrollo tardío del lenguaje implica comprender el fenómeno como parte del proceso de escolarización. Por lo tanto, la escuela no solo ofrece al niño el aprendizaje formal del sistema de escritura sino que también un nuevo contexto comunicativo: el 'lenguaje académico' el que posee exigencias comunicativas y cognitivas propias.

En segundo lugar, esta propuesta permite comprender la gramática no como estructuras aisladas sino, más bien, como recursos clave que el sujeto debe aprender a utilizar estratégicamente para la construcción y comprensión de diversos discursos. De este modo, la gramática es concebida como un repertorio de posibilidades. En este sentido, se hace necesario entender el funcionamiento de la complejidad sintáctica en el lenguaje académico ligada a los modos de representación y organización de la información (Snow \& Uccelli, 2009).

En tercer lugar, la visión del lenguaje propuesta por Bajtín permite describir los diversos eventos comunicativos no solo en términos de las modalidades comunicativas. En efecto, se ha asociado, principalmente, a la escuela con el espacio de la escritura; sin embargo, la propuesta de géneros primarios y secundarios permite comprender desde una perspectiva multidimensional todos los factores que están en juego en la comunicación dentro de la escuela. Por lo tanto, desde una mirada instruccional, se podría establecer relaciones entre oralidad y escritura académica, ofreciendo oportunidades de desarrollar discursos particulares con mayores exigencias desde el punto de vista epistémico, gramatical y léxico. De 
esta manera, se rompe la dicotomía entre oralidad y escritura propia del pensamiento ilustrado, visión que afianza la estructura por sobre la actividad realizada por los sujetos a través del lenguaje.

Por último, cabe destacar que la visión centrada en los géneros discursivos y en los discursos permite poner en el centro de la reflexión no solo la dimensión comunicativa del lenguaje, sino también la relación con la actividad cognitiva. "Al (re)producir un tipo de discurso, el agente debe proceder a la planificación interna de los segmentos en cuestión, aprendiendo así a ejecutar esos procesos indisolublemente mentales y verbales como son los razonamientos" (Bronckart, 2008, 16). Al enseñar explícitamente discursos más exigentes, no solo se les da oportunidades a los estudiantes de aprender a comunicarse adecuadamente en un contexto comunicativo formal, sino que también se le da la posibilidad de aprender los modos de razonamiento de determinadas disciplinas.

\section{REFERENCIAS BIBLIOGRÁFICAS}

ÁLVAREZ, Gerardo (2006): "Un enfoque discursivo/textual para la enseñanza de idiomas", en Onomázein, 14(2), 163-169.

BAJTÍN, Mijail (1982): Estética de la creación verbal. México DF: Siglo XXI.

BAZERMAN, Charles (2005) "An essay on pedagogy by Mikhail M. Bakhtin", en Written Communication, 22(3), 333-338.

BERMAN, Ruth, \& KATZENBERGER, Irit (2004): "Form and function in introducing narrative and expository texts: A developmental perspective", en Discourse Processes, 38(1), 57-94.

BERMAN, Ruth (2008): "The psycholinguistics of developing text construction", en Journal of Child Language, 35(4), 735-771.

BERMAN, Ruth \& NIR-SAGIV, Bracha (2007): "Comparing narrative and expository text construction across adolescence: A developmental paradox", en Discourse Processes, 43(2), 79-120.

BRONCKART, Jean Paul (2008): "Actividad lingüística y construcción de conocimientos", en Lectura y Vida, 2(29), 6-18.

BYRNES, James \& WASIK, Barbara (2009): "The development of grammatical knowledge", en BYRNES \& WASIK (Eds.), Language and literacy development. What educators need to know (pp. 131-168). New York, NY: The Guilford Press.

CHARAUDEAU, Patrick \& MAINGUENEAU, Dominique (2005): Diccionario de análisis del discurso. Madrid: Amorrortu editores.

COLOMA, Carmen, PEÑALOZA, Cristian. \& FERNÁNDEZ, Reyes (2007): "Producción de oraciones complejas en niños de 8 y 10 años", en RLA. Revista de Lingüistica Teórica y Aplicada, 45(1), 33-44.

CUBERO, Mercedes \& SÁNCHEZ, Jose (2002): "Práctica social y modos de hablar y pensar", en Infancia y Aprendizaje, 25(1), 101-117. 
EMERSON, Carl (1993): "La palabra externa y el habla interna: Bajtín, Vigostki y la internalización", en MORSON (Ed.), Bajtín. Ensayos y diálogos sobre su obra. México DF: El estudio.

HUNT, Kellogg (1968): Grammatical structures written at three grade levels. Urbana, IL: National Council of Teachers of English.

LOBAN, Walter (1976): Language development: Kindergarten through grade twelve. Urbana, IL: National Council of Teachers of English.

LÓPEZ GARCÍA, Angel (2000): "Relaciones paratácticas e hipotácticas", en BOSQUE \& DEMONTE (Eds.), Gramática descriptiva de la lengua española (Vol. 3, pp. 3509-3547). Madrid: Espasa Calpe.

MEDINA, Lorena (2002): "¿Para qué aprender gramática en la escuela? Puentes entre la abstracción del análisis y la comunicación cotidiana", en Onomázein, 7, 183-212.

NIPPOLD, Marilyn (2004): "Research on later language development. International perspectives", en BERMAN (Ed.), Language development across childhood and adolescence (pp. 1-8). Philadelphia, PA: John Benjamins Publishing.

NIR, Bracha \& BERMAN, Ruth (2010): "Complex syntax as a window on contrastive rhetoric", en Journal of Pragmatics, 42(3), 744-765.

RAVID, Dorit \& TOLCHINSKY, Liliana (2002): "Developing linguistic literacy: a comprehensive model", en Journal of Child Language, 29(2), 417-447.

SCHLEPPEGRELL, Mary (2001): "Linguistic features of the language of schooling", en Linguistics and Education, 12(4), 431-459.

SEBASTIÁN, Eugenia (2003): "Los inicios del lenguaje infantil sin conocimiento gramatical", en Cognitiva, 15(2), 207-213.

SILVESTRI, Adriana (2002a): "Comunicación y cognición en los modelos sociogenéticos", en Psykhe, 11(1), 109-115.

SILVESTRI, Adriana (2002b): "Funciones psicológicas y adquisición discursiva", en Propuesta Educativa, 25, 1-7.

SNOW, Catherine \& UCCELLI, Paula (2009): "The Challenge of Academic Language", en OLSON \& TORRANCE (Eds.), The Cambridge Handbook of Literacy (pp. 112-133). New York: Cambridge University Press.

STEWART, Susan (1993): "Gritos en la calle: la antilingüística de Bajtín", en MORSON (Ed.), Bajtín. Ensayos y diálogos sobre su obra. México DF: El estudio.

TOLCHINSKY, Liliana \& SIMÓ, Rosa (2001): Escribir y leer a través del currículum. Barcelona: Cuadernos de Educación.

TOMASELLO, Michael (2000): "First steps towards a usage-based theory of language acquisition", en Cognitive Linguistics, 11(1/2), 61-82.

VALENCIA, Alba, ÁVILA, Emilio, MUÑOZ, Gloria, NÚÑEZ, Nicolás, ECHEVERRÍA, Max \& VÉLIZ, Mónica (1991): "Evaluación de la madurez 
sintáctica en estudiantes chilenos de cuarto medio", en Estudios Filológicos, 71 82.

VOLOSHINOV, Valentín \& BAJTÍN, Mijail (1998): "La construcción de la enunciación", en VOLOSHINOV \& BAJTÍN (Eds.), ¿Qué es el lenguaje? (pp. 43-78). Buenos Aires: Editorial Almagesto.

VYGOTSKY, Lev (1979): El desarrollo de los procesos psicológicos superiores. Barcelona: Editorial Crítica.

VÉLIZ, Mónica (1988): "Evaluación de la madurez sintáctica en el discurso escrito", en RLA Revista de Lingüística Teórica y Aplicada, 26, 105-114.

VÉLIZ, Mónica (1999): "Complejidad sintáctica y modo de discurso", en Estudios Filológicos, 34, 181-192. 